\title{
A nested cohort study of 6,248 early breast cancer patients treated in neoadjuvant and adjuvant chemotherapy trials investigating the prognostic value of chemotherapy- related toxicities
}

Jean E. Abraham ${ }^{1,2,3,5^{*}+}$, Louise Hiller ${ }^{6{ }^{*}+}$, Leila Dorling ${ }^{3+}$, Anne-Laure Vallier ${ }^{4,5}$, Janet Dunn ${ }^{6}$, Sarah Bowden ${ }^{7}$, Susan Ingle ${ }^{1,2,4}$, Linda Jones ${ }^{1,2,5}$, Richard Hardy ${ }^{1,2,4}$, Christopher Twelves ${ }^{9}$, Christopher J. Poole ${ }^{6}$, Paul D P Pharoah ${ }^{3}$, Carlos Caldas ${ }^{1,2,5,8}$ and Helena M. Earl ${ }^{1,2,5}$

\begin{abstract}
Background: The relationship between chemotherapy-related toxicities and prognosis is unclear. Previous studies have examined the association of myelosuppression parameters or neuropathy with survival and reported conflicting results. This study aims to investigate 13 common chemotherapy toxicities and their association with relapse-free survival and breast cancer-specific survival.
\end{abstract}

Methods: Chemotherapy-related toxicities were collected prospectively for 6,248 women with early-stage breast cancer from four randomised controlled trials (NEAT; BR9601; tAnGo; Neo-tAnGo). Cox proportional-hazards modelling was used to analyse the association between chemotherapy-related toxicities and both breast cancer-specific survival and relapse-free survival. Models included important prognostic factors and stratified by variables violating the proportional hazards assumption.

Results: Multivariable analysis identified severe neutropenia (grades $\geq 3$ ) as an independent predictor of relapse-free survival (hazard ratio $(H R)=0.86 ; 95 \%$ confidence interval $(C l), 0.76-0.97 ; P=0.02)$. A similar trend was seen for breast cancer-specific survival ( $\mathrm{HR}=0.87 ; 95 \% \mathrm{Cl}, 0.75-1.01 ; P=0.06)$. Normal/low BMl patients experienced more severe neutropenia ( $P=0.008$ ) than patients with higher BMI. Patients with fatigue (grades $\geq 3$ ) showed a trend towards reduced survival (breast cancer-specific survival: $\mathrm{HR}=1.17 ; 95 \% \mathrm{Cl}, 0.99-1.37 ; P=0.06$ ). In the NEAT/BR9601 sub-group analysis by treatment component, this effect was statistically significant $(H R=1.61 ; 95 \% \mathrm{Cl}, 1.13-2.30 ; P=0.009)$.

Conclusions: This large study shows a significant association between chemotherapy-induced neutropenia and increased survival. It also identifies a strong relationship between low/normal BMI and increased incidence of severe neutropenia. It provides evidence to support the development of neutropenia-adapted clinical trials to investigate optimal dose calculation and its impact on clinical outcome. This is important in populations where obesity may lead to sub-optimal chemotherapy doses.

Keywords: Adverse events, Breast cancer, Chemotherapy, Prognosis, Survival, Toxicity

\footnotetext{
* Correspondence: ja344@medschl.cam.ac.uk; l.hiller@warwick.ac.uk

${ }^{\dagger}$ Equal contributors

'Department of Oncology, Addenbrooke's Hospital, University of Cambridge,

Hills Road, Box 193, Cambridge CB2 OQQ, UK

${ }^{6}$ Warwick Clinical Trials Unit, University of Warwick, Gibbet Hill Road,

Coventry CV4 7AL, UK

Full list of author information is available at the end of the article
} 


\section{Background}

Chemotherapy-related toxicities (CRTs) are a common complication of treatment in all cancers. For each CRT, multiple factors contribute to their development, including pharmacogenetic and co-morbidity factors [1]. The relationship between the occurrence of various CRTs and subsequent survival has been investigated in relatively small cohorts in multiple tumour types with conflicting results. A CRT may be a proxy pharmacokinetic parameter, indicating the level of drug exposure, dose density delivered and/or metabolic activity, or it may be a proxy pharmacodynamic parameter that reflects the sensitivity and susceptibility of different tissues to chemotherapy.

Many studies in different tumour types have investigated the association between survival and measures of myelosuppression. Eskander et al. [2] reviewed seven breast cancer studies with inter-study heterogeneity in trial design and varying toxicities, including leukocyte nadir, myelosuppression and neutropenia. The largest study [3] $(\mathrm{n}=750)$, showed that patients with grade 2 or 3 neutropenia, on the National Cancer Institute Common Toxicity Criteria for Adverse Events (NCI CTCAE) scale, had a $10 \%$ absolute survival advantage at 5 years compared to those with no neutropenia (multivariable $P=0.037$ ). Shitara et al. [4] performed a meta-analysis of 13 trials $(n=9,528)$ considering several different toxicities, varying tumour types, stages of disease, and thresholds of NCI CTCAE classification and concluded that neutropenia or leukopenia experienced during chemotherapy was associated with improved survival.

The association between survival and taxane-related sensory neuropathy in breast cancer patients has been explored previously. Schneider et al. [5] investigated 4,554 patients from a randomised controlled clinical trial and found no significant relationship between neuropathy and disease-free survival (DFS), overall survival, or relapse-free survival (RFS). However, Moreno-Aspitia et al. [6] did report an association of taxane-related sensory neuropathy with DFS in early stage, taxanetreated, human epidermal growth factor (HER2)-positive breast cancer patients. In ovarian cancer, Lee et al. [7] found that sensory neuropathy secondary to treatment with paclitaxel and carboplatin was associated with improved progression-free survival $(n=949)$.

Moderate and/or severe oral mucositis was associated with improved survival in one study [8] $(n=533)$. Another study associated oral mucositis with an increased risk of infection and an adverse impact on survival [9].

Although there is considerable data on the impact of fatigue on quality of life $[10,11]$ in early stage breast cancer, there is no published evidence on the prognostic significance of chemotherapy-induced fatigue in early stage disease.
We have investigated the association between 13 common CRTs and RFS and breast cancer-specific survival (BCSS) in patients $(n=6,248)$ with early stage breast cancer using data from randomised controlled trials with prospective protocol-driven collection of CRTs.

\section{Methods}

\section{Patients and clinical trials}

Clinical data was collected from the UK randomised clinical trials NEAT $(\mathrm{n}=2027)$ [12], BR9601 $(\mathrm{n}=374)$ [12], tAnGo $(\mathrm{n}=3152)$ [13], and Neo-tAnGo $(\mathrm{n}=831)$ [14], creating a nested cohort of 6,248 patients, from a total of 6,384 patients, included in this study after providing adequate quality toxicity data. Additional file 1: Figure S1a,b summarises the individual clinical trials included and their trial objectives. Table 1 summarises patient characteristics of the 6,248 patients, with Additional file 1: Table S1 showing patient characteristics by each trial. Median follow-up was 6.2 years, with 1,335 (21 \%) breast cancer-related events, 148 (2\%) non-breast cancer-related deaths, and 4,765 (77\%) live patients. For the analysis of RFS, there were 1,888 events (30\%) recorded and 4,360 (70\%) censored observations. Written informed consent was obtained from each patient recruited into the trials. All the trials involved received full ethical approval from a UK ethical review board and completed all other regulatory requirements prior to commencement.

\section{Phenotypes}

In all trials, CRTs were evaluated during each chemotherapy cycle for each patient. CRTs were graded using NCI CTCAE (version 2 or 3; Table S2 in Additional file 1) by the investigators at the participating centres and data collected centrally via case report forms. For each of the 13 CRTs of interest (Fig. 1 - Consort diagram for chemotherapy-related toxicity analyses), patients were categorised into a case or control based on their maximum reported grade of the CRT throughout their chemotherapy treatment (Table S3, Additional file 1). All trials required pre-treatment blood count assessment prior to administration of each cycle and neutropenia was classified from immediate pre-chemotherapy blood tests. Blood draw was avoided during the expected white blood cell nadir period.

\section{Ethics, consent and permissions}

Ethical approval was obtained for tAnGo (West Midlands: 00/7/44), Neo-tAnGo (South East: 04/ MRE01/60), NEAT/BR9601 (West Midlands: 30/04/ 1996) and PGSNPS (Cambridgeshire: 05Q0108/71).

All patients gave their consent to participate in the trials. 
Table 1 Summary of patient characteristics for the study cohort

Study cohort
$\mathrm{n}$

Randomised treatment
E-CMF
CMF
EC-T
EC-TG
T-EC
TG-EC
Age, years
$\leq 50$
$>50$
ER status

$\begin{array}{ll}\text { Negative } & 2551 \\ \text { Positive } & 3591 \\ \text { Missing } & 106 \\ \text { GR status } & \end{array}$

Negative 2463

Positive 2652

Missing 1133

HER2 status

Negative

3760

Positive

1034

Missing

Nodal status

Negative

1-3 positive

Clinically negative, neoadjuvant

Clinically positive, neoadjuvant

4+ positive

$1156 \quad 19$

1149

1773

1769

200

201

3606

2642

2551

3591

106

463

Breast cancer-specific survival

Breast cancer related deaths

Deaths due to other cause

Alive

Relapse-free survival

Events

Censored

1454

1366

2383

409

403

1687

1335

148

4765

1888

4360

Triple negative status

No (ER ${ }^{+}$and HER2-)

Yes (ER', $\mathrm{PGR}^{-}$or unknown, and HER2-)

1242

Missing

2685

ECOG performance status

0

$\geq 1$

Missing

Table 1 Summary of patient characteristics for the study cohort (Continued)

\begin{tabular}{lll}
\hline Tumour size, mm & & \\
$0-20$ & 2195 & 35 \\
$21-50$ & 3375 & 54 \\
$>50$ & 475 & 8 \\
Missing & 203 & 3 \\
Tumour grade & & \\
1 & 146 & 2 \\
2 & 2206 & 35 \\
3 & 3654 & 59 \\
Missing & 242 & 4 \\
Menopausal status & & \\
Pre/peri & 3480 & 56 \\
Post & 2200 & 35 \\
Missing & 568 & 9 \\
BMl & & \\
Underweight $(<18.5)$ & 69 & 1 \\
Healthy weight $(18.5$ to $<25)$ & 2503 & 40 \\
Overweight $(25$ to $<30)$ & 2088 & 33 \\
Obese $(\geq 30)$ & 1469 & 24 \\
\hline
\end{tabular}

ER Estrogen receptor; $p G R$ Progesterone receptor; HER2 Human epidermal growth factor receptor; ECOG Eastern Co-operative Oncology Group; BMI Body mass index; $E$ Epirubicin; $C$ Cyclophosphamide; $M$ Methotrexate; $F 5$-fluouroucil; $T$ Paclitaxel; $G$ Gemcitabine

\section{Statistical analysis}

To investigate the association between CRTs and outcomes, BCSS time was calculated from date of treatment cessation to date of death due to breast cancer, or to date of death due to other causes, or date of censoring in women still alive. RFS time was calculated from date of treatment cessation to either the date of first relapse or date of death in women dying without relapse, or to date of censoring for those alive and relapse free.

Cox proportional-hazards modelling was used to investigate the association between CRTs experienced (categorised as shown in Additional file 1: Table S3) and BCSS and RFS. A base Cox model was created by testing the association of important prognostic factors with BCSS and RFS. Any factors which were significant at $P$ $<0.05$ in univariable analysis were entered into a multivariable Cox model and factors remaining significant on adjustment in the multivariable model were retained for the base Cox model. The proportional hazards assumption was checked using the Schoenfeld residuals method [15]. Subsequent models were stratified by variables that violated the proportional hazards assumption. All CRTs significant in a univariable Cox model at the $P<0.1$ level were entered into the multivariable base Cox model to assess their association with BCSS and RFS. Associations 


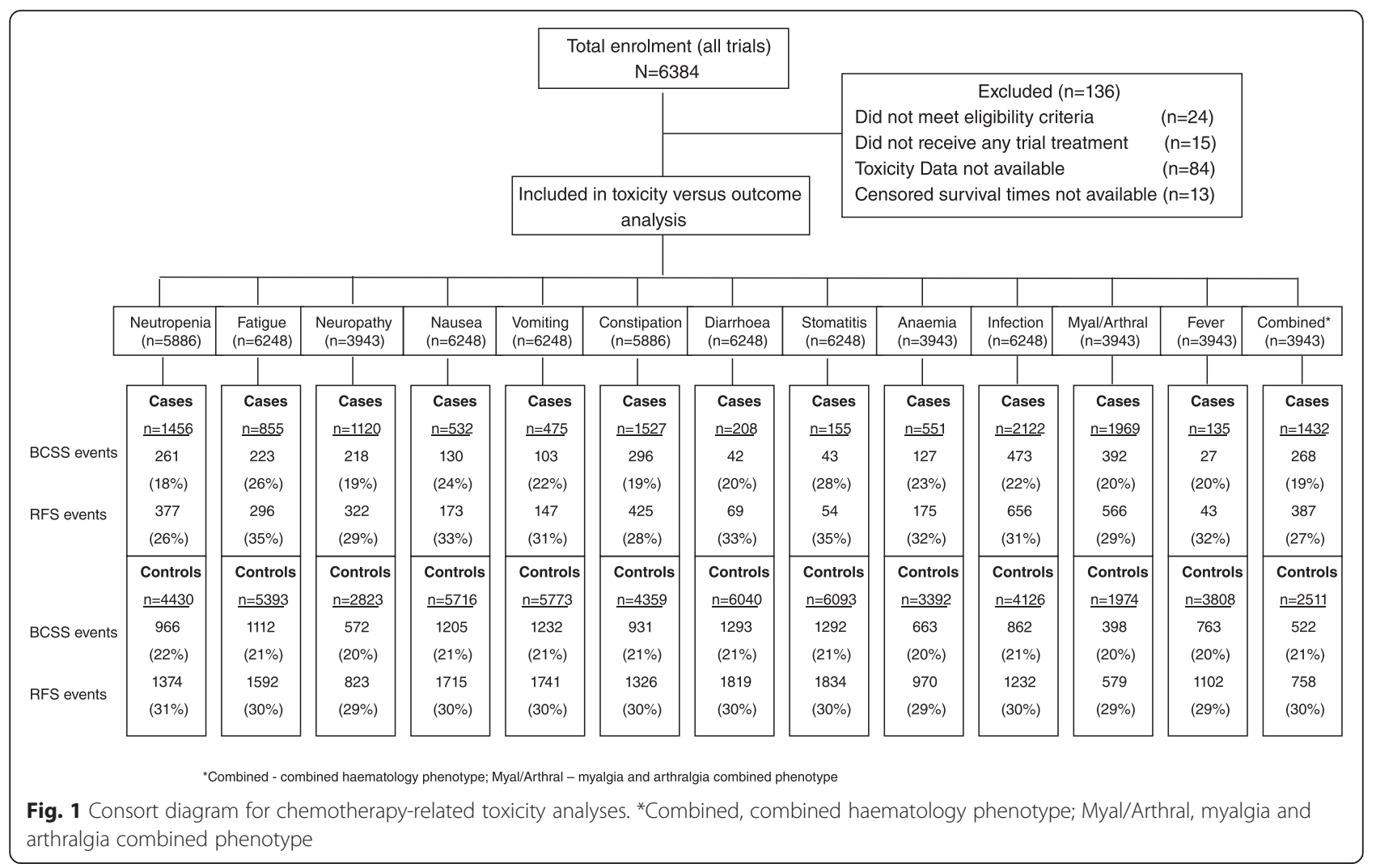

between CRTs and BCSS or RFS were deemed statistically significant if the $P$ value was $<0.05$.

Any CRT showing a relationship with outcome at this stage was further investigated. To determine if the relationship found was independent of dose intensity (DI) (sub-optimal DI $(<85 \%)$ versus optimal DI $(>85 \%)$ ) [16], the analysis was re-run adjusting for DI. Due to the known relationship between increasing BMI and poor prognosis $[17,18]$, we similarly assessed if adjusting for BMI affected the relationship between the CRT and RFS/BCSS. Additionally, CRT relationships with known prognostic factors were assessed using $X^{2}$ tests with continuity corrections.

After performing this analysis on all 6,248 patients, seven different components of the treatment regimens received by the group of patients were investigated (Table S4 in Additional file 1), including (1) epirubicin (E); (2) cyclophosphamide, methotrexate and 5fluorouracil (CMF) after having received $\mathrm{E}$; (3) CMF as the sole treatment regimen; (4) EC as a primary component; (5) paclitaxel (T) and/or gemcitabine (G) after receiving EC; (6) $\mathrm{T}$ and/or $\mathrm{G}$ as a primary component; and (7) EC after having received $\mathrm{T}$ and/or G). Case-control re-classification for each of the CRTs of interest was undertaken focusing purely on the patients' maximum reported grade during the different components of their particular treatment regimen.
Association with increased or decreased RFS and BCSS was assessed.

\section{Results}

\section{Analysis of maximum CRT across all chemotherapy} treatments

The base Cox model included trial, performance status (PS) and nodal status, and was stratified by tumour size, tumour grade and estrogen receptor (ER) status. Neutropenia, fatigue, anaemia, combined haematological toxicity and constipation were nominally significant $(P<0.1)$ for either BCSS or RFS (or both) on univariable analysis (Table 2). All other CRTs were not found to be associated with either RFS or BCSS. After adjustment for other prognostic factors in the model, only neutropenia was significant. Fatigue was not statistically significant after adjustment (BCSS; $\mathrm{HR}=1.17 ; 95 \% \mathrm{CI}, 0.99-1.37 ; P=0.06)$.

\section{Neutropenia}

Neutropenia status was available for 5,886 patients, of whom $1,456(25 \%)$ recorded neutropenia grade $\geq 3$ over the course of their entire chemotherapy treatment; 4,430 (75 \%) did not. After adjusting for the base model prognostic factors, neutropenia remained a statistically significant, independent predictor of RFS (HR $=0.86 ; 95 \%$ CI, 0.76-0.97; $P=0.02$ ), with a similar trend seen for 
Table 2 Analysis of maximum CRT across all treatments

\begin{tabular}{|c|c|c|c|c|c|c|c|c|c|c|}
\hline \multirow[t]{3}{*}{ Toxicity } & \multirow{3}{*}{$\begin{array}{l}\mathrm{n} \\
\text { (Univariable } \\
\text { analysis) }\end{array}$} & \multirow{3}{*}{$\begin{array}{l}\mathrm{n} \\
\text { (Multivariable } \\
\text { analysis) }\end{array}$} & \multicolumn{4}{|l|}{ BCSS } & \multicolumn{4}{|l|}{ RFS } \\
\hline & & & \multicolumn{2}{|l|}{ Unadjusted } & \multicolumn{2}{|l|}{ Adjusted } & \multicolumn{2}{|l|}{ Unadjusted } & \multicolumn{2}{|l|}{ Adjusted } \\
\hline & & & $\begin{array}{l}\mathrm{HR} \\
(95 \% \mathrm{Cl})\end{array}$ & $P$ value & $\begin{array}{l}\mathrm{HR} \\
(95 \% \mathrm{Cl})\end{array}$ & $P$ value & $\begin{array}{l}\mathrm{HR} \\
(95 \% \mathrm{Cl})\end{array}$ & $P$ value & $\begin{array}{l}\mathrm{HR} \\
(95 \% \mathrm{Cl})\end{array}$ & $P$ value \\
\hline Neutropenia $^{\mathrm{a}}$ & 5886 & 5211 & $\begin{array}{l}0.85 \\
(0.74-0.98)\end{array}$ & 0.02 & $\begin{array}{l}0.87 \\
(0.75-1.01)\end{array}$ & 0.06 & $\begin{array}{l}0.85 \\
(0.76-0.95)\end{array}$ & 0.004 & $\begin{array}{l}0.86 \\
(0.76-0.97)\end{array}$ & 0.02 \\
\hline Nausea & 6248 & 5468 & $\begin{array}{l}1.11 \\
(0.93-1.33)\end{array}$ & 0.25 & $\begin{array}{l}1.09 \\
(0.90-1.33)\end{array}$ & 0.37 & $\begin{array}{l}1.07 \\
(0.91-1.25)\end{array}$ & 0.42 & $\begin{array}{l}1.06 \\
(0.90-1.26)\end{array}$ & 0.47 \\
\hline Vomiting & 6248 & 5468 & $\begin{array}{l}0.98 \\
(0.80-1.19)\end{array}$ & 0.80 & $\begin{array}{l}1.03 \\
(0.84-1.28)\end{array}$ & 0.76 & $\begin{array}{l}1.00 \\
(0.85-1.18)\end{array}$ & $>0.99$ & $\begin{array}{l}1.05 \\
(0.88-1.26)\end{array}$ & 0.57 \\
\hline Stomatitis & 6248 & 5468 & $\begin{array}{l}1.20 \\
(0.89-1.63)\end{array}$ & 0.24 & $\begin{array}{l}1.25 \\
(0.90-1.74)\end{array}$ & 0.19 & $\begin{array}{l}1.09 \\
(0.83-1.43)\end{array}$ & 0.54 & $\begin{array}{l}1.12 \\
(0.83-1.50)\end{array}$ & 0.46 \\
\hline Constipation & 5886 & 5211 & $\begin{array}{l}0.91 \\
(0.80-1.04)\end{array}$ & 0.17 & $\begin{array}{l}0.95 \\
(0.82-1.09)\end{array}$ & 0.45 & $\begin{array}{l}0.91 \\
(0.82-1.01)\end{array}$ & 0.09 & $\begin{array}{l}0.94 \\
(0.83-1.05)\end{array}$ & 0.27 \\
\hline Diarrhoea & 6248 & 5468 & $\begin{array}{l}0.93 \\
(0.68-1.26)\end{array}$ & 0.64 & $\begin{array}{l}0.98 \\
(0.70-1.38)\end{array}$ & 0.93 & $\begin{array}{l}1.08 \\
(0.85-1.37)\end{array}$ & 0.54 & $\begin{array}{l}1.19 \\
(0.92-1.55)\end{array}$ & 0.18 \\
\hline Infection & 6248 & 5468 & $\begin{array}{l}1.09 \\
(0.97-1.22)\end{array}$ & 0.14 & $\begin{array}{l}1.01 \\
(0.90-1.15)\end{array}$ & 0.82 & $\begin{array}{l}1.06 \\
(0.96-1.16)\end{array}$ & 0.26 & $\begin{array}{l}1.01 \\
(0.91-1.12)\end{array}$ & 0.88 \\
\hline Fatigue & 6248 & 5468 & $\begin{array}{l}1.24 \\
(1.07-1.43)\end{array}$ & 0.004 & $\begin{array}{l}1.17 \\
(0.99-1.37)\end{array}$ & 0.06 & $\begin{array}{l}1.17 \\
(1.03-1.32)\end{array}$ & 0.01 & $\begin{array}{l}1.13 \\
(0.99-1.30)\end{array}$ & 0.08 \\
\hline Anaemia & 3943 & 3582 & $\begin{array}{l}1.18 \\
(0.97-1.42)\end{array}$ & 0.09 & $\begin{array}{l}1.14 \\
(0.93-1.39)\end{array}$ & 0.21 & $\begin{array}{l}1.11 \\
(0.95-1.30)\end{array}$ & 0.20 & $\begin{array}{l}1.08 \\
(0.91-1.28)\end{array}$ & 0.36 \\
\hline $\begin{array}{l}\text { Combined } \\
\text { haematological }\end{array}$ & 3943 & 3582 & $\begin{array}{l}0.89 \\
(0.77-1.03)\end{array}$ & 0.11 & $\begin{array}{l}0.88 \\
(0.76-1.03)\end{array}$ & 0.12 & $\begin{array}{l}0.88 \\
(0.78-0.99)\end{array}$ & 0.03 & $\begin{array}{l}0.88 \\
(0.78-1.00)\end{array}$ & 0.06 \\
\hline Neurotoxicity & 3943 & 3582 & $\begin{array}{l}0.96 \\
(0.82-1.13)\end{array}$ & 0.64 & $\begin{array}{l}0.99 \\
(0.84-1.17)\end{array}$ & 0.90 & $\begin{array}{l}0.98 \\
(0.86-1.12)\end{array}$ & 0.78 & $\begin{array}{l}0.99 \\
(0.87-1.14)\end{array}$ & 0.94 \\
\hline Myalgia & 3943 & 3582 & $\begin{array}{l}0.95 \\
(0.82-1.09)\end{array}$ & 0.43 & $\begin{array}{l}1.03 \\
(0.89-1.20)\end{array}$ & 0.69 & $\begin{array}{l}0.95 \\
(0.84-1.06)\end{array}$ & 0.34 & $\begin{array}{l}1.02 \\
(0.91-1.16)\end{array}$ & 0.70 \\
\hline Fever & 3943 & 3582 & $\begin{array}{l}0.98 \\
(0.67-1.44)\end{array}$ & 0.91 & $\begin{array}{l}0.84 \\
(0.57-1.26)\end{array}$ & 0.41 & $\begin{array}{l}1.08 \\
(0.80-1.46)\end{array}$ & 0.63 & $\begin{array}{l}0.98 \\
(0.71-1.34)\end{array}$ & 0.88 \\
\hline
\end{tabular}

BCSS Breast cancer-specific survival; RFS Relapse-free survival; HR Hazard ratio; Cl Confidence interval

${ }^{a}$ Cases classified as National Cancer Institute Common Toxicity Criteria for Adverse Events (NCl CTCAEAE) grade $\geq 3$

BCSS $(\mathrm{HR}=0.87 ; 95 \% \mathrm{CI}, 0.75-1.01 ; P=0.06)$. The association was strengthened after further adjustment for DI (BCSS: $\mathrm{HR}=0.85 ; 95 \% \mathrm{CI}, 0.73-0.98 ; P=0.03$; RFS: $H R=0.84 ; 95 \%$ CI, 0.74-0.95; $P=0.005$ ). Patients with grade $\geq 3$ neutropenia were likely to survive and remain relapse free for longer when compared to patients who experienced grades $\leq 2$. Neutropenia appeared unrelated to triple negative status $(P=0.85)$, ER status $(P=0.46)$, and HER2 status $(P=0.36)$.

Underweight and normal BMI patients were more likely to record severe neutropenia during their treatment course than overweight or obese BMI patients (27\% vs. $24 \%, P=0.008$ ). Given the known association of increasing BMI with poor prognosis $[17,18]$, we repeated the analysis adjusting for BMI, to confirm that the relationship between neutropenia and RFS/BCSS was independent of BMI. Adjusting for BMI only marginally changed the results (BCSS: $\mathrm{HR}=0.88 ; 95 \% \mathrm{CI}$, 0.76-1.02; $P=0.08$; RFS: $\mathrm{HR}=0.87 ; 95 \% \mathrm{CI}, 0.77-0.98$; $P=0.02$ ). Adjustment for both DI and BMI simultaneously resulted in similar findings to when adjusting only for DI (BCSS: $\mathrm{HR}=0.85 ; 95 \% \mathrm{CI}, 0.74-0.99 ; P=0.04$; RFS: $\mathrm{HR}=0.84 ; 95 \% \mathrm{CI}, \quad 0.74-0.95 ; \quad P=0.006)$. Underweight and normal patients were also more likely to report moderate-severe vomiting (grade $\geq 2, P=0.009$ ). Obese and overweight patients recorded more diarrhoea (grade $\geq 2, P=0.02$ ), infection (grade $\geq 2, P=0.005$ ), neuropathy (grade $\geq 2, \quad P<0.0001$ ) and arthralgia/myalgia (grade $\geq 2, P=0.002$ ), but these CRTs were not associated with either improved or reduced survival.

Older patients and post-menopausal patients were more likely to have neutropenia during their treatment course ( $28 \%$ vs. $22 \%, P<0.0001$ and $28 \%$ vs. $22 \%, P<0.0001$, respectively).

The extent of neutropenia experienced by the study cohort was not influenced by the use of prophylactic growth colony stimulating factor (GCSF). GCSF was not routinely given as prophylaxis as part of the trial protocol of any of the study trials. GCSF use was allowed secondary to an admission for febrile neutropenia and/or based on clinical 
judgement. However, only in $9 \%$ of patients was the use of GCSF ever reported. It is unlikely, therefore, that this would have a significant impact on the overall results shown.

As previous studies [19] have classified neutropenia as no neutropenia versus any (grade 0 vs. $\geq 1$ ), we repeated the neutropenia analysis using this classification (Table 3). The multivariable analysis demonstrated a statistically significant association between neutropenia and BCSS ( $\mathrm{HR}=0.87 ; 95 \% \mathrm{CI}, 0.77-0.99 ; P=0.03)$ with a similar trend for RFS (HR $=0.91 ; 95 \% \mathrm{CI}, 0.82-1.01 ; P=0.07)$.

\section{Fatigue}

Fatigue status was available for 6,248 patients, of whom 855 (14\%) recorded fatigue grades $\geq 3$ at some point in their chemotherapy treatment whereas 5,393 (86 \%) did not. Fatigue was associated with poorer BCSS and RFS in the univariable models, but the associations were attenuated and no longer significant after adjusting for other prognostic variables (BCSS: $\mathrm{HR}=1.17$; $95 \% \mathrm{CI}, 0.99-1.37 ; P=0.06$; RFS: $\mathrm{HR}=1.13 ; 95 \% \mathrm{CI}, 0.99-1.30 ; P=0.08)$.

Fatigue appeared unrelated to BMI $(P=0.76)$, triple negative status $(P=0.50)$, HER2 status $(P=0.86)$, age $(P=0.33)$, menopausal status $(P=0.27)$, and GCSF administration $(P=0.89)$. ER negative patients may be more likely to be classed as a fatigue case during their treatment course (15\% vs. $13 \%$ of ER positive patients, $P=0.06$ ), although this was not statistically significant at the $P=0.05$ threshold and may not be of clinical significance.

\section{CRTs of interest during specific chemotherapy regimen components}

In order to identify if CRTs reported during particular combination chemotherapy treatments were associated with increased or decreased RFS and BCSS, the analysis was repeated considering only the maximum $\mathrm{NCI}$ CTCAE grade documented during a specific chemotherapy regimen, rather the maximum NCI CTCAE grade across all chemotherapy treatments (Additional file 1: Table S4).

\section{Neutropenia}

Patients who experienced neutropenia grade $\geq 3$ whilst receiving epirubicin and cyclophosphamide (EC) as their first chemotherapy component (drug regimen 4) were significantly more likely to survive and remain relapse free than those who did not (BCSS: $\mathrm{HR}=0.83 ; 95 \% \mathrm{CI}$, 0.69-1.00; $P=0.05$; RFS: HR $=0.85 ; 95 \%$ CI, 0.73-0.99; $P=0.04$ ) (Additional file 1: Table S4). However, experiencing neutropenia grade $\geq 3$ whilst receiving $T \pm G$ as their second chemotherapy component (having already received EC, drug regimen 5) was not significantly associated with outcome (BCSS: $\mathrm{HR}=1.07 ; 95 \% \mathrm{CI}$, 0.83-1.37; $P=0.62$; RFS: HR $=1.00 ; 95 \% \mathrm{CI}, 0.80-1.24$; $P=0.99$ ).

Neutropenia grade $\geq 3$ being reported either during $\mathrm{T} \pm \mathrm{G}$ as a first chemotherapy component, or during $\mathrm{EC}$ as the second chemotherapy component (after $\mathrm{T} \pm \mathrm{G}$ ) was not significantly associated with outcome (drug regimens 6 and 7), although the numbers of patients in these datasets were small ( $n=270$ and 260, respectively).

Furthermore, when drug regimens 4 (CRT recorded in $\mathrm{EC}$, as the first chemotherapy) and 7 (CRT recorded in $\mathrm{EC}$, as the second chemotherapy after $\mathrm{T} \pm \mathrm{G}$ ) were combined to create a sample consisting of all tAnGo and Neo-tAnGo patients receiving EC at any cycle of their treatment, severe neutropenia was significantly associated with both increased BCSS $(\mathrm{HR}=0.83 ; 95 \% \mathrm{CI}$, $0.69-0.99 ; P=0.04)$ and RFS (HR $=0.84 ; 95 \% \mathrm{CI}, 0.73-$ $0.98 ; P=0.03)$. Neutropenia was not a significant predictor of survival for tAnGo and Neo-tAnGo patients when it occurred during $\mathrm{T} \pm \mathrm{G}$ regimens.

\section{Fatigue}

Patients experiencing moderate-severe fatigue whilst receiving epirubicin (E) as their first chemotherapy component (drug regimen 1) had significantly worse outcomes (BCSS: $\mathrm{HR}=1.48 ; 95 \% \mathrm{CI}, 1.03-2.12 ; P=0.03$; RFS: $\mathrm{HR}=$ 1.34; $95 \% \mathrm{CI}, 0.97-1.85) ; P=0.07)$ than those who did not record moderate-severe fatigue. Similarly, patients reporting severe fatigue during the period they received CMF as their second chemotherapy component (after completing E; drug regimen 2), had reduced BCSS ( $\mathrm{HR}=$ 1.61; $95 \% \mathrm{CI}, 1.13-2.30 ; P=0.009)$ and RFS (HR = 1.39; $95 \% \mathrm{CI}, 1.01-1.92 ; P=0.05)$. Interestingly, this strong effect was not seen for NEAT and BR9601 patients receiving CMF only (drug regimen 3). Fatigue was not a significant predictor of survival for tAnGo and Neo-tAnGo patients on $\mathrm{EC}$ and $\mathrm{T} \pm \mathrm{G}$ (Additional file 1: Table S4: drug regimens 4-7).

Table 3 Analysis of neutropenia across all treatments (classification National Cancer Institute Common Toxicity Criteria for Adverse Events (NCI CTCAE) grade $\geq 1$ vs. grade 0 )

\begin{tabular}{|c|c|c|c|c|c|c|c|c|c|c|}
\hline \multirow[t]{3}{*}{ Toxicity } & \multirow{3}{*}{$\begin{array}{l}\mathrm{n} \\
\text { (univariable } \\
\text { analysis) }\end{array}$} & \multirow{3}{*}{$\begin{array}{l}\mathrm{n} \\
\text { (multivariable } \\
\text { analysis) }\end{array}$} & \multicolumn{4}{|l|}{ BCSS } & \multicolumn{4}{|l|}{ RFS } \\
\hline & & & \multicolumn{2}{|l|}{ Unadjusted } & \multicolumn{2}{|l|}{ Adjusted } & \multicolumn{2}{|l|}{ Unadjusted } & \multicolumn{2}{|l|}{ Adjusted } \\
\hline & & & $\mathrm{HR}(95 \% \mathrm{Cl})$ & $P$ value & $\mathrm{HR}(95 \% \mathrm{Cl})$ & $P$ value & $\mathrm{HR}(95 \% \mathrm{Cl})$ & $P$ value & $\mathrm{HR}(95 \% \mathrm{Cl})$ & $P$ value \\
\hline Neutropenia & 5,886 & 5,211 & $0.86(0.77-0.96)$ & 0.009 & $0.87(0.77-0.99)$ & 0.03 & $0.91(0.83-0.99)$ & 0.04 & $0.91(0.82-1.01)$ & 0.07 \\
\hline
\end{tabular}

BCSS Breast cancer-specific survival; RFS Relapse-free survival; HR Hazard ratio; Cl, Confidence interval 


\section{Discussion}

We have investigated the association between 13 CRTs and clinical outcome (RFS and BCSS) in 6,248 patients with early breast cancer treated within randomised clinical trials of neoadjuvant and adjuvant chemotherapy. The majority of CRTs were not associated with either RFS or $\mathrm{BCSS}$. However, we have demonstrated that severe neutropenia (grades $\geq 3$ ) is associated with improved RFS. In addition, after re-classification of neutropenia case status to grades $\geq 1$, the association with BCSS remains. Previous studies investigating the relationship between neutropenia and survival have hypothesised that neutropenia is a reflection of chemotherapy efficacy and activity. This implies that patients who are not achieving neutropenia may also not be receiving an effective or adequately active dose [18]. It must be noted that, in this study, 13 separate CRTs have been tested against two clinical endpoints of BCSS and RFS and, as such, the study findings must be considered whilst bearing in mind the issue of multiple testing.

Patients with normal or underweight BMI are more likely to have severe neutropenia during their treatment course in comparison to overweight or obese BMI patients $(P=0.008)$. In obese patients, due to an increase in the amount of fat contributing to the actual weight and potentially changes in blood flow, the pharmacokinetics of chemotherapy may be affected. This may affect volume of distribution, clearance, and, consequently, patient drug exposure. Thus, overweight or obese BMI patients may not be receiving an adequate dose, although further investigations would be required to confirm this. The disadvantages of using body surface area (BSA) to dose patients have been discussed at length elsewhere [20-22]. Drug disposition can show 4-10-fold inter-individual variability, which is inadequately compensated for by using BSA. There is a strong argument to include other variables to allow more accurate dose estimation for each individual.

Bergh et al. [23] conducted a randomised trial comparing high-dose chemotherapy versus haematologically-tailored adjuvant chemotherapy to assess the effect on RFS and overall survival. The haematologically-tailored arm specified that participants experienced pre-defined levels of haematological toxicities. This study demonstrated that tailored chemotherapy rather than high-dose chemotherapy showed improved RFS. One shortcoming of this study was that it used high-dose chemotherapy as the 'control' arm, which is not the current standard treatment for early breast cancer. Lindeman et al. [24] conducted a trial using haematological criteria for selecting dosing strategy. Study results, available in abstract form only, did not show a statistically significant improvement in survival with tailored-chemotherapy compared with standard BSA-based chemotherapy. However, both distant
DFS and DFS showed trends towards a better outcome for the tailored chemotherapy arm. Our results show that chemotherapy-induced neutropenia is an additional prognostic factor for longer-term outcomes, and we suggest that this could be tested in trials randomising between personalising chemotherapy within an adaptive protocol and standard chemotherapy dosing. Interestingly, trials in which doses of cyclophosphamide and doxorubicin were increased (NSABP B22 [25], NSABP B25 [26], and CALGB 9344 [27]) did not show benefit in long-term outcomes. However, dose-dense trials which increased the frequency of chemotherapy dosing with filgrastim support (CALGB 9741 [28] and a 10-study meta-analysis [29]) have shown improvement in longer-term outcomes. The authors of CALGB 9741 stated that it was their impression that the improvements seen in CALGB 9741 were as a result of the more frequent administration of chemotherapy, and that use of filgrastim did not by itself add to the efficacy of dose-dense treatment [30].

These trials have applied dose intensification with filgrastim support, which has abrogated any 'signal' from neutropenia; in fact, they have usually shown less febrile neutropenia and neutropenic sepsis in the dose-dense arm. In our study, we have looked at the prognostic effect of developing neutropenia in trials which have used standard chemotherapy dosing without routine filgrastim support. Future trials would need to establish whether adapting doses to achieve neutropenia would improve outcomes. Our hypothesis is that host factors (such as pharmacodynamic and pharmacogenomic factors) influence the level of chemotherapy-induced neutropenia, and only those patients who do not achieve neutropenia with standard doses may benefit from intensification of chemotherapy. The evidence would suggest that adapting to a dose-dense protocol may be the most effective way to intensify chemotherapy. The results show that patients achieving grade $\geq 1$ have a statistically significant survival advantage. This allows the possibility of using a simple standard haematological measurement to adjust dose, whereby dose is adjusted until neutropenia NCI CTCAE grade $\geq 1$ is achieved, after which treatment would be maintained at the same dosing level.

The potential mechanisms that may explain why chemotherapy-induced neutropenia is associated with improved survival include neutropenia as a marker of cancer stem cell death [22, 31]. Other studies have proposed that neutrophils may be involved in the control of the microenvironment in sites of metastatic spread [32-34]. Chia et al. [35] comment that toxicity and clinical outcome may be more likely to correlate when the therapeutic agent targets the biological driver of the disease directly, for example, sunitinib-associated hypertension [36]. Although the mechanism behind the association between chemotherapy-induced neutropenia and clinical outcome 
is unclear, this study, in conjunction with previously published data [2-4], provides strong evidence that this association is real and clinically relevant.

Moderate-severe fatigue (NCI CTCAE $\geq 2$ ) may be associated with a reduced BCSS. This effect was significant in the univariable analysis of fatigue across all treatments $(\mathrm{HR}=1.24 ; 95 \% \mathrm{CI}, 1.07-1.43 ; P=0.004)$ but on multivariable analysis BCSS (HR $=1.17$; $95 \%$ CI, 0.99$1.37 ; P=0.06$ ) became less significant. However, analysis of the treatment regimens showed that there was a statistically significant association between moderatesevere fatigue and BCSS in patients treated with ECMF (Additional file 1: Table S4). The patient characteristics across all the trials is similar, although $20 \%$ of patients in NEAT and BR9601 had fatigue classified as grade $\geq 2$, in comparison to only $10 \%$ in tAnGo and Neo-tAnGo. It is notable that $18 \%$ of NEAT and BR9601 had a pre-treatment PS $\geq 1$, in comparison to only $8 \%$ and $4 \%$ of tAnGo and Neo-tAnGo patients, respectively (Additional file 1: Table S1). Trial eligibility criteria required that patients must have a PS $\leq 2$. It is unclear whether this difference in baseline PS alone accounts for the increased levels of moderate-severe fatigue seen in NEAT and BR9601; however, reduced pre-treatment PS would be likely to increase the risk of severe fatigue during treatment $[10,11]$.

\section{Conclusions}

This large and comprehensive study has shown a statistically significant association between improved survival and neutropenia (using toxicity classification NCI CTCAE $\geq 1$ or $\geq 3$ ). This association is clinically relevant and has the potential to be further tested in neutropenia-adapted treatment regimens within clinical trials to assess its potential to improve clinical outcome. This study shows that patients with normal or reduced BMI experience greater rates of neutropenia in comparison to overweight and obese patients. This is particularly relevant in populations where increasing levels of obesity may mean that a significant proportion of breast cancer patients are receiving sub-optimal chemotherapy doses. This study also indicates that chemotherapy-induced fatigue may be an indicator of poor clinical outcome. Patients' pre-treatment performance status needs to be adequately assessed and levels of treatment-induced fatigue need to be carefully monitored and moderated.

\section{Availability of data and materials}

Data supporting these findings is held by the Trial Management Group for PGSNPS study, where the original concept for this analysis was designed. Any access requires appropriate ethical approvals and would be assessed by the Trial Management Group which includes the respective Chief Investigators of the clinical trials and PGSNPS, and would require a specific Data Transfer Agreement.

\section{Additional file}

Additional file 1: Table S1. Patient characteristics of the 6,248 patients shown by trial. Table S2. National Cancer Institute Common Toxicity Criteria for Adverse Events (NCI CTCAE) version 2. Table S3. Case-control classification method for the 13 CRTs investigated. Table S4. Neutropenia and fatigue in relation to outcome, split by different treatment components. Figure S1. Trial objectives, outcomes and treatment regimens of the contributing clinical trials. (DOCX $82 \mathrm{~kb})$

\section{Competing interests}

The following part of the trial funding was received from pharmaceutical companies: Neo-tAnGo funding: Additional unrestricted educational grant from Eli Lilly Limited who also provided free Gemzar /gemcitabine. Bristol Myers Squibb Ltd provided free Taxol\%/paclitaxel from January 2005 to June 2006. tAnGo funding: Unrestricted educational grants and free drug from Eli Lilly (Gemzar ${ }^{\mathrm{TM}}$ ) and Bristol Myers Squibb (Taxol ${ }^{\mathrm{TM}}$ ). NEAT/BR9601 funding: 1996-2003: Unrestricted educational grant Pfizer (formerly Pharmacia). The authors declare that they have no other competing interests.

\section{Authors' contributions}

Chief Investigators of the trials/studies involved in this research: HME (NeotAnGo/NEAT); CJP (tAnGo/NEAT); CT (BR9601); JEA (PGSNPS). JEA, LH and LD designed the study. JEA, LH, SB, RH, SI, ALV, CT, CJP, and HME coordinated the trial and data collection. LH, LD and JEA analysed the data. JEA, LD, LH, PDPP, HME, and CC interpreted the data and contributed to manuscript writing. PDPP, CC and HE are joint senior authors. All authors reviewed the manuscript and approved the final version for publication. JEA, PDPP, CC, and $\mathrm{HE}$ were responsible for overall co-ordination of PGSNPS.

\section{Acknowledgements}

The initial background work for this study was completed in patients recruited to the PGSNPS pharmacogenetics study. We would like to like to thank all the women who participated in PGSNPS, NEAT, BR9601, tAnGo and Neo-tAnGo. Also, thanks to the clinical teams who recruited patients to all these studies. All authors read and approved the final version of the manuscript for submission.

\section{Financial support}

The funding bodies had no role in the design or conduct of the study, or decision to submit the manuscript for publication. PGSNPS funding: Cancer Research UK (project/fellowship grant numbers: C507/A6306 and C10097/ A7484). Neo-tAnGo funding: Cancer Research UK Research Grant (C57) A4180). Additional unrestricted educational grant from Eli Lilly Limited who also provided free Gemzar /gemcitabine. Bristol Myers Squibb Ltd provided free Taxol /paclitaxel from January 2005 to June 2006 (EudraCT No: 2004002356-34; ISRCTN 78234870; ClinicalTrials.gov number: NCT00070278). tAnGo funding: Unrestricted educational grants and free drug from Eli Lilly $\left(\right.$ Gemzar $\left.^{T M}\right)$ and Bristol Myers Squibb (Taxol ${ }^{\text {TM }}$ ). NEAT/BR9601 funding: project grant from Cancer Research UK (formerly Cancer Research Campaign) 19962003: Unrestricted educational grant Pfizer (formerly Pharmacia). HME, JEA, CC acknowledge funding from the NIHR Cambridge Biomedical Research Centre. JEA and LD acknowledge funding from Addenbrookes Charitable Trust. LD acknowledges funding from Medical Research Council.

\section{Author details}

${ }^{1}$ Department of Oncology, Addenbrooke's Hospital, University of Cambridge, Hills Road, Box 193, Cambridge CB2 0QQ, UK. ${ }^{2}$ NIHR Cambridge Biomedical Research Centre and Cambridge Experimental Cancer Medicine Centre, Box 277Hills Road, Cambridge CB2 0QQ, UK. ${ }^{3}$ Strangeways Research Laboratory, University of Cambridge, 2 Worts Causeway, Cambridge CB1 8RN, UK. ${ }^{4}$ Department of Oncology, Cambridge Cancer Trials Centre, Box 279 (S4)Addenbrooke's Hospital, Cambridge CB2 0QQ, UK. ${ }^{5}$ Cambridge Breast Unit and Cambridge University Hospitals NHS Foundation Trust, Hills Road, Cambridge CB2 OQQ, UK. ${ }^{6}$ Warwick Clinical Trials Unit, University of Warwick, 
Gibbet Hill Road, Coventry CV4 7AL, UK. ${ }^{7}$ Cancer Research UK Clinical Trials Unit, Institute for Cancer Studies, University of Birmingham, Edgbaston Birmingham B15 2TT, UK. ${ }^{8}$ Cancer Research UK Cambridge Institute, University of Cambridge, Li Ka Shing Centre, Robinson Way, Cambridge CB2 ORE, UK. ${ }^{9}$ Level 4, Leeds Institute of Cancer and Pathology and Leeds Experimental Cancer Medical Centre, St James Institute of Oncology, Beckett Street, Leeds LS9 7TF, UK.

\section{Received: 28 September 2015 Accepted: 17 December 2015}

\section{Published online: 29 December 2015}

\section{References}

1. Abraham JE, Guo Q, Dorling L, Tyrer J, Ingle S, Hardy R, et al. Replication of genetic polymorphisms reported to be associated with taxane-related sensory neuropathy in patients with early breast cancer treated with paclitaxel. Clin Cancer Res Am Assoc Cancer Res Inc. 2014;20:2466-75.

2. Eskander RN, Tewari KS. Impact of chemotherapy-induced neutropenia on survival in patients with breast, ovarian and cervical cancer: a systematic review. J Hematol Malignancies. 2012;2:63-73.

3. Cameron DA, Massie C, Kerr G, Leonard RCF. Moderate neutropenia with adjuvant CMF confers improved survival in early breast cancer. Br J Cancer. 2003;89:1837-42

4. Shitara K, Matsuo K, Oze I, Mizota A, Kondo C, Nomura M, et al. Metaanalysis of neutropenia or leukopenia as a prognostic factor in patients with malignant disease undergoing chemotherapy. Cancer Chemother Pharmacol. 2011;68:301-7.

5. Schneider BP, Zhao F, Wang M, Stearns V, Martino S, Jones V, et al. Neuropathy is not associated with clinical outcomes in patients receiving adjuvant taxanecontaining therapy for operable breast cancer. J Clin Oncol. 2012;30:3051-7.

6. Moreno-Aspitia A, Dueck A, Patel T, Hillman D, Tenner K, Dakhil S, et al. Paclitaxel-related peripheral neuropathy associated with improved outcome of patients with early stage HER2+ breast cancer who did not receive trastuzumab in the N9831 clinical trial. Cancer Res. 2009;69:2100.

7. Lee CK, Gurney H, Brown C, Sorio R, Donadello N, Tulunay G, et al. Carboplatinpaclitaxel-induced leukopenia and neuropathy predict progression-free survival in recurrent ovarian cancer. Br J Cancer. 2011;105:360-5.

8. McTiernan A, Jinks RC, Sydes MR, Uscinksa B, Hook JM, van Glabbeke M, et al. Presence of chemotherapy-induced toxicity predicts improved survival in patients with localised extremity osteosarcoma treated with doxorubicin and cisplatin: a report from the European Osteosarcoma Intergroup. Euro J Cancer. 2012:48:703-12

9. Lalla RV, Sonis ST, Peterson DE. Management of oral mucositis in patients with cancer. Dent Clin North Am. 2008:52:61-5.

10. Berger AM, Lockhart K, Agrawal S. Variability of patterns of fatigue and quality of life over time based on different breast cancer adjuvant chemotherapy regimens. Oncol Nurs Forum. 2009;36:563-70.

11. So WK, Marsh G, Ling WM, Leung FY, Lo JC, Yeung $M$, et al. The symptom cluster of fatigue, pain, anxiety, and depression and the effect on the quality of life of women receiving treatment for breast cancer: a multicenter study. Oncol Nurs Forum. 2009;36:E205-14.

12. Poole CJ, Earl HM, Hiller L, Dunn JA, Bathers S, Grieve R, et al. Epirubicin and cyclophosphamide, methotrexate, and fluorouracil as adjuvant therapy for early breast cancer. N Engl J Med. 2006;355:1851-62.

13. Poole CJ, Hiller L, Howard HC, Dunn JA, Canney P, Wardley AM, et al. tAnGo trial collaborators. tAnGo: A randomized phase III trial of gemcitabine (gem) in paclitaxel containing, epirubicin/cyclophosphamide-based, adjuvant chemotherapy (CT) for women with early-stage breast cancer (EBC). J Clin Oncol. 2008:26:15S. abstract 506.

14. Earl HM, Vallier A-L, Hiller L, Fenwick N, Young J, Iddawela M, et al. Effects of the addition of gemcitabine, and paclitaxel-first sequencing, in neoadjuvant sequential epirubicin, cyclophosphamide, and paclitaxel for women with high-risk early breast cancer (Neo-tAnGo): an open-label, $2 \times 2$ factorial randomised phase 3 trial. Lancet Oncol. 2014;15:201-12.

15. Hess KR. Graphical methods for assessing violations of the proportional hazards assumption in Cox regression. Stat Med. 1995;14:1707-23.

16. Earl HM, Hiller L, Dunn JA, Bathers S, Harvey P, Stanley A, et al. NEAT: National Epirubicin Adjuvant Trial-toxicity, delivered dose intensity and quality of life. Br J Cancer. 2008;99:1226-31.

17. Chan DSM, Vieira AR, Aune D, Bandera EV, Greenwood DC, McTiernan A, et al. Body mass index and survival in women with breast cancer-systematic literature review and meta-analysis of 82 follow-up studies. Ann Oncol. 2014; 25(10):1901-14. doi:10.1093/annonc/mdu042.

18. Sinicrope FA, Dannenberg AJ. Obesity and breast cancer prognosis: weight of the evidence. J Clin Oncol. 2011:29:4-7.

19. Di Maio M, Gridelli C, Gallo C, Shepherd F, Piantedosi FV, Cigolari S, et al. Chemotherapy-induced neutropenia and treatment efficacy in advanced non-small-cell lung cancer: a pooled analysis of three randomised trials. Lancet Oncol. 2005;6:669-77.

20. Gurney $H$. Dose calculation of anticancer drugs: a review of the current practice and introduction of an alternative. J Clin Oncol. 1996;14:2590-611.

21. Gurney H. I don't underdose my patients ... do I? Lancet. 2005:6:637-8.

22. Bergh J, Wiklund T, Erikstein B, Fornander T, Bengtsson NO, Malmström P, et al. Dosage of adjuvant G-CSF (filgrastim)-supported FEC polychemotherapy based on equivalent haematological toxicity in high risk breast cancer patients. Ann Oncol. 1998:9:403-11.

23. Bergh J, Wiklund T, Erikstein B, Lidbrink E, Lindman $H$, Malmström $P$, et al. Tailored fluorouracil, epirubicin, and cyclophosphamide compared with marrow-supported high-dose chemotherapy as adjuvant treatment for high-risk breast cancer: a randomised trial. Lancet. 2000;356:1384-91.

24. Lindman $H$, Andersson $M$, Edlund $P$, Bjerre KD, Hatschek $T$, Mouridsen HT, et al. A randomized study of individually tailored toxicity-based dosage of fluorouracil-epirubicin-cyclophosphamide chemotherapy (FEC) for early breast cancer. J Clin Oncol. 2011;29 Suppl:abstract 1017.

25. Fisher B, Anderson S, Wickerham DL, DeCillis A, Dimitrov N, Mamounas E, et al Increased intensification and total dose of cyclophosphamide in a doxorubicin-cyclophosphamide regimen for the treatment of primary breast cancer: findings from National Surgical Adjuvant Breast and Bowel Project B-22. J Clin Oncol. 1997:15:1858-69.

26. Wolmark N, Fisher B, Anderson S. The effect of increasing dose intensity and cumulative dose of adjuvant cyclophosphamide in node positive breast cancer: results of NSABP B-25. Breast Cancer Res Treat. 1997:46:26

27. Henderson IC, Berry DA, Demetri GD, Cirrincione CT, Goldstein L, Martino S, et al. Improved outcomes from adding sequential Paclitaxel but not from escalating Doxorubicin dose in an adjuvant chemotherapy regimen for patients with node-positive primary breast cancer. J Clin Oncol. 2003;21:976-83

28. Citron ML, Berry DA, Cirrincione C, Hudis C Winer EP Gradishar WJ, et al. Randomized trial of dose-dense versus conventionally scheduled and sequential versus concurrent combination chemotherapy as postoperative adjuvant treatment of node-positive primary breast cancer: first report of intergroup trial C9741. Cancer and Leukemia Group B Trial 9741. J Clin Oncol. 2003:21(8):1431-9.

29. Bonilla L, Ben-Aharon I, Vidal L, Gafter-Gvili A, Leibovici L, Stemmer SM. Dose-dense chemotherapy in nonmetastatic breast cancer: a systematic review and meta-analysis of randomized controlled trials. J Natl Cancer Inst. 2010;102:1845-54.

30. Citron ML, Berry DA, Cirrincione C, Hudis C, Norton L, Winer EP, et al. In Reply regarding: Randomized trial of dose-dense versus conventionally scheduled and sequential versus concurrent combination chemotherapy as postoperative adjuvant treatment of node-positive primary breast cancer: first report of intergroup trial C9741. Cancer and Leukemia Group B Trial 9741. J Clin Oncol. 2004:22(4):751-3.

31. Rocconi RP, Matthews KS, Kemper MK, Hoskins KE, Barnes MN. Chemotherapy-related myelosuppression as a marker of survival in epithelial ovarian cancer patients. Gynecol Oncol. 2008;108:336-41.

32. Kowanetz M, Wu X, Lee J, Tan M, Hagenbeek T, Qu X, et al. Granulocyte-colony stimulating factor promotes lung metastasis through mobilization of Ly6G + Ly6C+ granulocytes. Proc Natl Acad Sci U S A. 2010;107:21248-55.

33. Houghton AM, Rzymkiewicz DM, Ji H, Gregory A, Egea EE, Metz H, et al. Neutrophil elastase-mediated degradation of IRS-1 accelerates lung tumor growth. Nat Med. 2010;16:219-23.

34. Nozawa H, Chiu C, Hanahan D. Infiltrating neutrophils mediate the initial angiogenic switch in a mouse model of multistage carcinogenesis. Proc Natl Acad Sci U S A. 2006:103:12493-8.

35. Chia SK, Gelmon K. Linking toxicity to the target. J Clin Oncol. 2012;30(25): 3039-40.

36. Rini BI, Cohen DP, Lu DR, Chen I, Hariharan S, Gore M, et al. Hypertension as a biomarker of efficacy in-patients with metastatic renal cell carcinoma treated with sunitinib. J Natl Cancer Inst. 2011;103:763-73. 